\title{
Extrapolating B-H Curve Data using Common Electrical Steel Characteristics for High Magnetic Saturation Applications
}

\author{
Seung-Hee Chai ${ }^{1}$, Ji-Hyun Kim ${ }^{1}$, Sung-Il Kim ${ }^{2}$, and Jung-Pyo Hong ${ }^{1 *}$ \\ ${ }^{1}$ Department of Automotive Engineering, Hanyang University, Seoul 133-791, Korea \\ ${ }^{2}$ Motor R\&D Group, Digital Appliances, Samsung Electronics, Suwon 443-742, Korea
}

(Received 1 June 2015, Received in final form 23 June 2015, Accepted 7 July 2015)

\begin{abstract}
In this paper, an adequate $\mathrm{B}-\mathrm{H}$ curve extrapolation method is proposed and its reliability is verified through experiments. A method is developed to estimate the magnetic saturation induction from the density of the lamination core and electrical resistivity. The magnetic saturation induction of electrical steels measured using a vibration sample magnetometer are compared with the analytical results to validate the accuracy of the proposed estimation method. It is found that the predicted error in the magnetic saturation induction of the electrical steels are approximately $1.2 \%$ when the proposed method is used. The performance of interior permanent magnet synchronous motors that applies the proposed method are evaluated via $2 \mathrm{D}$ nonlinear finite element analysis and through experiments. Based on the obtained results, the extrapolated B-H curves from the estimated saturation induction can be used for various analyses in saturation region.
\end{abstract}

Keywords : B-H curve, electrical steel, extrapolation method, magnetic saturation induction

\section{Introduction}

In order to preserve fossil fuels and prevent further global warming, the demands for the development of vehicles that use electrical propulsion systems, such as hybrid electric vehicles and electric vehicles have increased. In particular, interior permanent magnet synchronous motors (IPMSMs) are employed for traction applications [1]. Indeed, IPMSMs have a higher torque density and efficiency per unit rotor volume than other types of motors, owing to the utilization of magnetic and reluctance torques. Hence, a wide operating speed range can be obtained with the help of field weakening control $[2,3]$.

Finite element analysis (FEA) is the most accurate method to predict performance of an electrical machine because it considers the nonlinear B-H properties of electrical steel used for laminating electrical machines. However, it is difficult to achieve appropriate rotor feature design that yields satisfactory results in terms of backelectromotive force (BEMF), average torque, etc. Fig. 1 illustrates an example of IPMSMs, which is designed to carry a high magnetic flux density. In general, electrical

CThe Korean Magnetics Society. All rights reserved.

*Corresponding author: Tel: $+82-2-2220-4466$

Fax:+82-2-2220-4465, e-mail: hongjp@hanyang.ac.kr
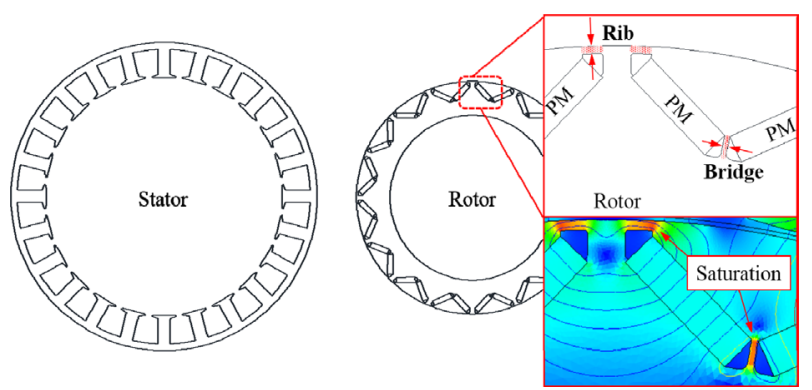

Fig. 1. (Color online) Saturation part (rib and bridge) of electric machines.

motors are designed to drive approximately $1.5 \mathrm{~T}$ (Tesla unit) magnetic flux density in normal conditions. However, in severe operating conditions, higher flux densities close to the saturation range can be required. In particular, the rotor's rib and bridge are designed in a manner to reduce leakage flux, as shown in Fig. 1. The latter is related to motor efficiency and output, and thus, the rib and bridge's magnetic flux densities are saturated over $2 \mathrm{~T}$. The problem becomes more apparent when fractional-slot concentrated windings are used for higher magnetic saturation in the rotor's core [4]. Hence, accurate B-H curve information is required for reliable analysis of the rotor's saturated parts.

Frequently, an Epstein frame is used in international 


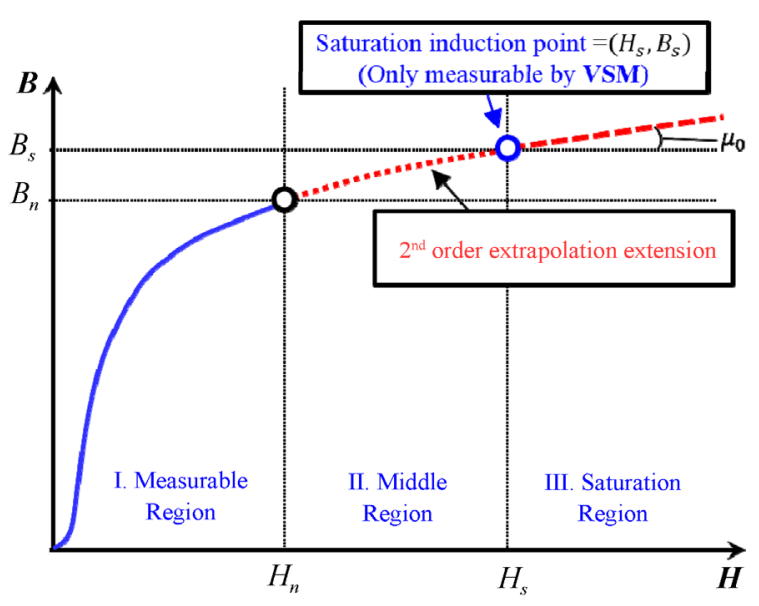

Fig. 2. (Color online) Extrapolate unmeasurable B-H data.

standards to measure B-H curves. However, this measurement system is limited to measuring B-H curves below saturation. Thus, the B-H curve can only be measured up to the point $B_{n}$, as shown in Fig. 2. The extrapolation technique is practical in extrapolating B-H data if magnetic flux density, $B_{s}$ can be determined [5]. However, the saturation value is not available to the users and can only be measured with a vibration sample magnetometer (VSM), or can be estimated from the chemical composition of the material. Unfortunately, the VSM equipment is not easily available for use because of its high cost and large size. Therefore, a measurement method is required in order to extrapolate the $\mathrm{B}-\mathrm{H}$ data from the magnetic saturation point without using the VSM equipment. It is important to appropriately use the extrapolation method in the saturated area of the B-H curve, and to exactly predict the required value of the magnetic saturation induction.

Few academic papers discuss the extrapolation method of B-H curves in order to define appropriate values for the parameters of the analytical curve. Typical extrapolation methods are introduced in [5]. The first method predicts the area beyond saturation by assuming that the slope at the last point of the measured data is the permeability value of space. The second method performs extrapolation on the area beyond saturation using the slope of the last two points of the measured data. Neither of these two methods can ensure the accuracy of the slope (permeability), calculated based on the measured data, nor can they predict an exact magnetic saturation induction value. The Law of Approach to Saturation (LAS) mentioned in $[18,19]$ performs extrapolation on the magnetic saturation induction as a polynomial series. The Exponential Law Extrapolation (ELE) method in [18] and [20] also performs extrapolation but as an exponential function.
However, the methods used in these two models does not derive exact prediction values of the magnetic saturation induction because the required constant values in calculation are estimated from the last two points of the data curve, measured using the Epstein frame. The Saturation Field Extrapolation (SFE), the method introduced in [18], predicts the magnetic saturation induction using both the last data value obtained before saturation, and a point in the saturated region. However, the method has a large range of error prediction because the prediction is based on a random value between the magnetic field $H_{s}^{\max }$ values obtained by the experiment and the magnetic field $H_{s}^{\min }$ values obtained by calculation. In this study, the B$\mathrm{H}$ data is extrapolated using the quadratic function, and a novel method is proposed to calculate the required magnetic saturation induction value $J_{s}$ from specific resistance and density values of the material. $J_{s}$ can be expressed according to the aluminum $(A l)$ and silicon $(S i)$ contents. These parameters are commonly provided in the catalogue of material properties [7]. In addition, the electrical resistivity and density of an electrical steel plate change according to the content of $\mathrm{Al}$ and Si. Many studies have been conducted to investigate these changes. However, they focused only on the relationship between the electrical resistivity and density of the electrical steel plate and the $\mathrm{Al}$ and $\mathrm{Si}$ contents [8,9]. In fact, few research studies have focused on the practical estimation of the magnetic saturation induction for electrical steel. If the relationships between the quadratic function, electrical resistivity, density, and metal content can be represented by a function, then $J_{s}$ can be calculated without using $A l$ and $\mathrm{Si}$ contents.

In order to verify the reliability of the proposed method for various electrical steels, the saturation induction values calculated by this method are compared to the average value of the repeatedly measured values for each specimen. In addition, the values of both BEMF and torque of the motor, which was manufactured by one of the specimens, are calculated by applying FEA, and are compared to the test values.

In summary, this paper presents a novel method that estimates the magnetic saturation induction using the values of the density of the lamination core and the electrical resistivity, which can be conveniently obtained through the derivation of a mathematical formula using the $\mathrm{Al}$ and $\mathrm{Si}$ contents (wt\%). Finally, the proposed method, which uses the quadratic equations to extrapolate from the magnetic saturation induction, is introduced and its applicability is discussed based on the result of a specimen test and a motor performance test. 


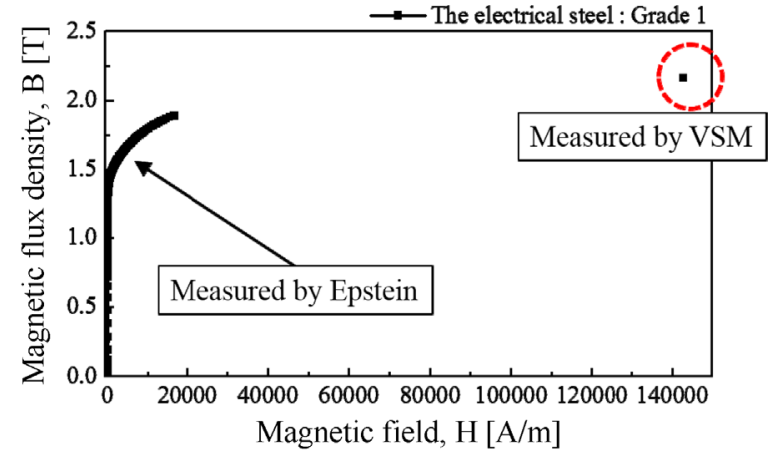

Fig. 3. (Color online) Measured B-H curve data of the representative electrical steel, Grade 1.

\section{Preliminary Measurements and Preparation}

In order to test the proposed extrapolation method, Epstein measurements are carried out for the five grades of electrical steel used as a representative soft magnetic material; these steels are commonly used in high power devices, with low, medium, or high $\mathrm{Si}$ and $\mathrm{Al}$ contents. The dimensions of the specimens are $3 \mathrm{~mm} \times 3 \mathrm{~mm}$, and the B-H curve data are averaged over five samples. The initial magnetization curve for electrical steel grade 1 is experimentally determined by the standard Epstein test, as illustrated in Fig. 3. To achieve the high magnetic field required for the validation of the data extrapolation, a VSM system is used to measure the magnetic saturation induction from the electrical steel grade 1 samples. The magnetic saturation inductions $J_{s}$ of the selected grades are tested at room temperature $(300 \mathrm{~K})$. To obtain the saturation state data, the VSM is excited with the specimens up to $80 \mathrm{kOe}(6,366 \mathrm{kA} / \mathrm{m})$, and the measured $\mathrm{B}-\mathrm{H}$ curves show saturation tendencies at $80 \mathrm{kOe}(6,366 \mathrm{kA} /$ $\mathrm{m})$, which brings the sample into saturation. The test results are discussed in Section 4 of this paper.

\section{Extrapolation of B-H Curve using the Quadratic Function}

\subsection{Extrapolation of $B-H$ curve using the quadratic} function

In this study, the curve is divided into three areas for proper extension of the B-H curve. As shown in Fig. 4, it is divided into a measurable Region I, an intermediate area Region II, and the saturated Region III that is open for investigation. Region $\mathrm{I}$ is a measurable area that includes the linear section and knee point, and the $B$ value can be measured based on the $H$ value using the Epstein equipment. Region II is an intermediate area that is defined as the middle region between the measurable area and the saturation area, where the relative permeability becomes equal to the relative permeability of air. Region III is the saturation area, where the permeability of the air is equal to the relative permeability of electrical steel. Equation (2) is used as the method for extrapolating Region III. The following linear function is used to estimate the saturation field flux density, while the quadratic extrapolation function is proposed to estimate the high field flux density in Region II [5]:

$$
\begin{aligned}
& H=a B^{2}+b B+c \\
& H=\left(B-J_{s}\right) / \mu_{0}
\end{aligned}
$$

Fig. 4 illustrates the B-H curve drawn based on equation (1), (2). In order to solve this equations, the boundary conditions for the points between the three areas have to be determined as shown in Fig. 4. In each boundary point of Region I-II and Region II-III, if the differential values for both areas before and after the boundary point are the same, then the following four conditions can be applied.

$$
H^{I}\left(B_{n}\right)=H^{I I}\left(B_{n}\right)
$$
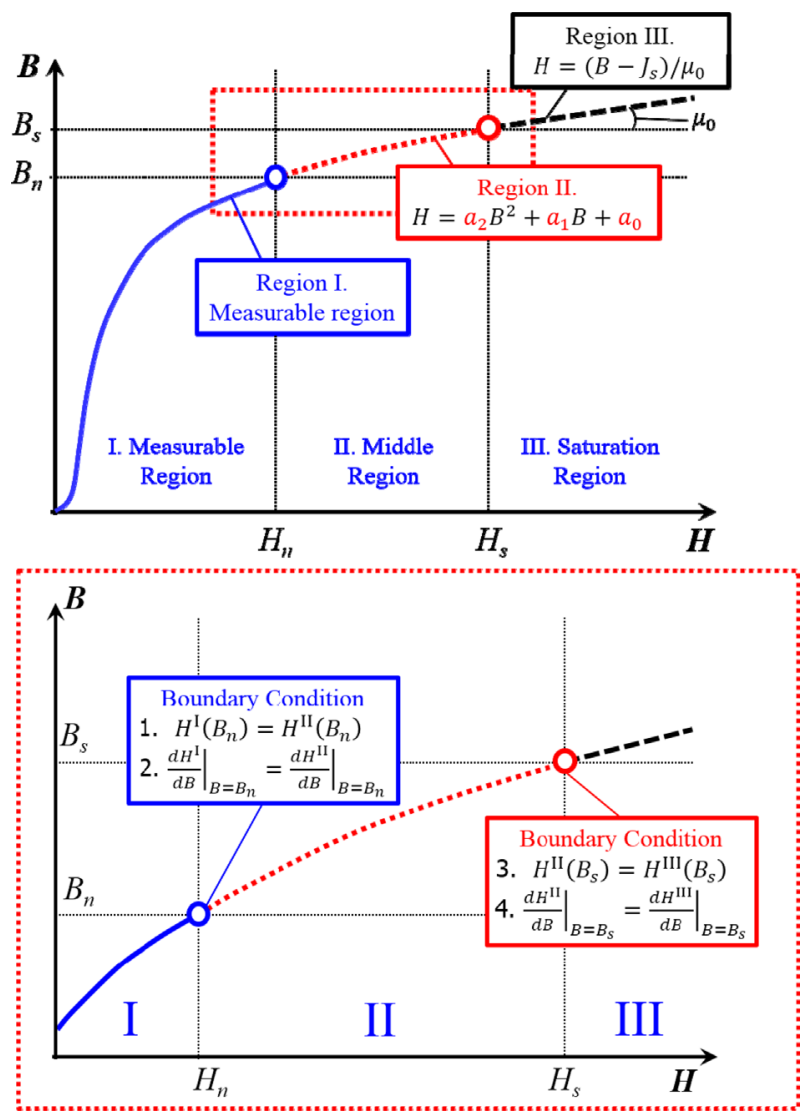

Fig. 4. (Color online) Method to extrapolate unmeasurable B$\mathrm{H}$ data and boundary conditions. 


$$
\begin{aligned}
& \left.\frac{d H^{I}}{d B}\right|_{B=B_{n}}=\left.\frac{d H^{I I}}{d B}\right|_{B=B_{n}} \\
& H^{I I}\left(B_{s}\right)=H^{I I I}\left(B_{s}\right) \\
& \left.\frac{d H^{I I}}{d B}\right|_{B=B_{s}}=\left.\frac{d H^{I I}}{d B}\right|_{B=B_{s}}
\end{aligned}
$$

Equations (7) to (10) can be obtained from equations (3) to (6) by applying measured data for Region I, equation (1) for Region II, and equation (2) for Region III. The four equations (7) to (10) can be solved with four unknowns, $a_{2}, a_{1}, a_{0}$, and $B_{s}$.

$$
\begin{aligned}
& a_{2} B_{n}^{2}+a_{1} B_{n}+a_{0}=H_{n} \\
& 2 a_{2} B_{n}+a_{1}=\mu_{n} \\
& a_{2} B_{s}^{2}+a_{1} B_{s}+a_{0}=\left(B_{s}-J_{s}\right) / \mu_{0} \\
& 2 a_{2} B_{s}+a_{1}=1 / \mu_{0}
\end{aligned}
$$

Where,

$$
\begin{aligned}
& \mu_{n}=\left(H_{n-1}-H_{n}\right) /\left(B_{n-1}-B_{n}\right) \\
& B_{s}=\left\{2\left(J_{s}+\mu_{0} H_{n}\right)-\left(1-\mu_{0} \mu_{n}\right) B_{n}\right\} /\left(1-\mu_{0} \mu_{n}\right) \\
& a_{2}=\left(1-\mu_{0} \mu_{n}\right) /\left\{2\left(B_{s}-B_{n}\right)\right\} \\
& a_{1}=\left(1 / \mu_{0}\right)-2 a_{2} B_{s} \\
& a_{0}=\left\{\left(B_{s}-J_{s}\right) / \mu_{0}\right\}-B_{s}\left(a_{2} B_{2}+a_{1}\right)
\end{aligned}
$$

Where, $J_{s}$ is the magnetic saturation induction, $\mu_{0}$ is the permeability of air, $H_{n}$ and $B_{n}$ are the $n$-th measured data of the field intensity and magnetic induction of the electrical steel, respectively. $H_{n-1}$ and $B_{n-1}$ are the corresponding $(n-1)$-th measured data. The $J_{s}$ is essential to realize an accurate extrapolation. This value can be obtained using the VSM. The system may be excited to over $80 \mathrm{kOe}(6,366 \mathrm{kA} / \mathrm{m})$ in a way that the electrical steel is saturated for the measurement of $J_{s}$. In general, this value cannot be measured without using the measurement equipment. Thus, a method is proposed to calculate $J_{s}$ from typical material data, which can be easily found in material catalogues.

\subsection{Estimation of magnetic saturation induction using} the material's characteristics

In order to estimate the $J_{s}$ value of a material, an empirical formula has been proposed under the condition that the weight percentage of alloy components such as $\mathrm{Al}$ and $\mathrm{Si}$ are known [10]. According to [11, 12], the following relation has been proposed:

$$
J_{s}=2.162-0.043 \times \% S i-0.0625 \times \% A l
$$

where $\% \mathrm{Al}$ and $\% \mathrm{Si}$ are the $\mathrm{Al}$ and $\mathrm{Si}$ contents. Although both direct measurement and estimation methods are acceptable owing to their high accuracy, few papers have reported the $J_{s}$ values of electrical steel and its alloy components. According to ASTM 677-07 [7], the density of the electrical steel can be determined from the $\mathrm{Al}$ and $\mathrm{Si}$ contents. Hence, the density determined from $\% \mathrm{Al}$ and $\% \mathrm{Si}$ shows a good agreement with the experimental results of our study. Moreover, the electrical resistivity can also be obtained through the empirically determined $\mathrm{Al}$ and $\mathrm{Si}$ material's contents [8]. The density of the material $d\left[\mathrm{~g} / \mathrm{cm}^{3}\right]$ and the electrical resistivity $R[\mu \Omega \cdot \mathrm{cm}]$ are given as follows:

$$
\begin{aligned}
& d=7.865-0.065(\% \mathrm{Si}+1.7 \times \% \mathrm{Al}) \\
& R=13+10.52 \times \% \mathrm{Si} \times 11.82+\% \mathrm{Al}
\end{aligned}
$$

From the equations (16) to (18), whose unknowns are the electrical steel's $\% \mathrm{Al}$ and $\% \mathrm{Si}$, and by combining these equations, the magnetic saturation induction $J_{s}[\mathrm{~T}]$ can be expressed as

$$
J_{s}=0.37863 \times d-0.00175 \times R-0.79318
$$

For convenience, $d$ and $R$ can be obtained from an electrical steel data sheet for different material grades.

With $J_{s}$, the parameters $B_{s}$ and $H_{s}$ can be estimated using equation (2), and the B-H curve can be extrapolated by the quadratic function (1).

\section{Experimental Results of the Extrapolation and Motor Characteristics}

\subsection{Validation of magnetic saturation induction and B-H curve extrapolation}

In the calculation, the density and resistance presented in the standard data of the electrical steel were used. The saturation induction $J_{s}$ values were calculated under each condition using the proposed equations (16) to (19). In order to verify the reliability of the proposed method, the calculated magnetic saturation induction and experimental values obtained using the VSM were compared. In the experiments, five grades of electrical steel were selected either with low, medium, or high $\mathrm{Si}$ and $\mathrm{Al}$ contents. The magnetic saturation inductions of the selected grades were tested using the VSM. The dimensions of the specimens and test conditions are given in Section 2 of this paper.

Table 1 shows that the calculated and experimental values of the magnetic saturation point $J_{s}$ have a gap ranging between $0.3 \%$ and $1.2 \%$, depending on the electrical steel specimen's type. As indicated above, the proposed formula is acceptable for the five grades containing different alloy components; hence, realizing minimal 
Table 1. Magnetic saturation induction comparison.

\begin{tabular}{cccccc}
\hline \hline Steel number & $\begin{array}{c}\text { Density } \\
\left(\mathrm{g} / \mathrm{cm}^{3}\right)\end{array}$ & $\begin{array}{c}\text { Resistivity } \\
(\mu \Omega \cdot \mathrm{cm})\end{array}$ & $\begin{array}{c}\text { Estimated } \boldsymbol{J}_{\boldsymbol{s}}, \\
(\mathbf{T})\end{array}$ & $\begin{array}{c}\text { Measured } \boldsymbol{J}_{\boldsymbol{s}}, \\
\mathbf{( T )}\end{array}$ & $\begin{array}{c}\text { Error } \\
(\boldsymbol{\%})\end{array}$ \\
\hline Grade 1 & 7.60 & 56.6 & $\mathbf{1 . 9 8 5}$ & $\mathbf{1 . 9 9 1}$ & $\mathbf{0 . 3}$ \\
Grade 2 & 7.65 & 48.6 & $\mathbf{2 . 0 1 8}$ & $\mathbf{2 . 0 1 4}$ & $\mathbf{0 . 2}$ \\
Grade 3 & 7.70 & 40.3 & $\mathbf{2 . 0 5 2}$ & $\mathbf{2 . 0 3 9}$ & $\mathbf{0 . 6}$ \\
Grade 4 & 7.80 & 25.3 & $\mathbf{2 . 1 1 6}$ & $\mathbf{2 . 1 1 5}$ & $\mathbf{0 . 4}$ \\
Grade 5 & 7.85 & 21.9 & $\mathbf{2 . 1 4 1}$ & $\mathbf{1 . 2}$ \\
\hline
\end{tabular}

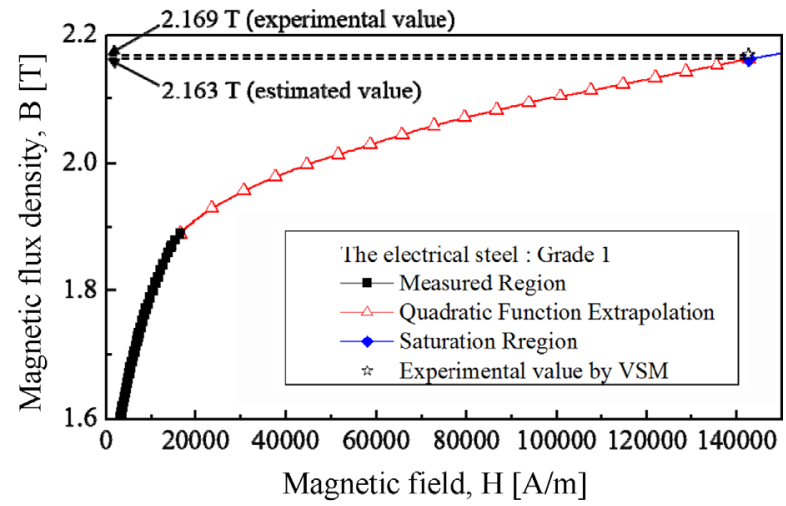

Fig. 5. (Color online) Extrapolated data of B-H curve for the representative electrical steel, Grade 1.

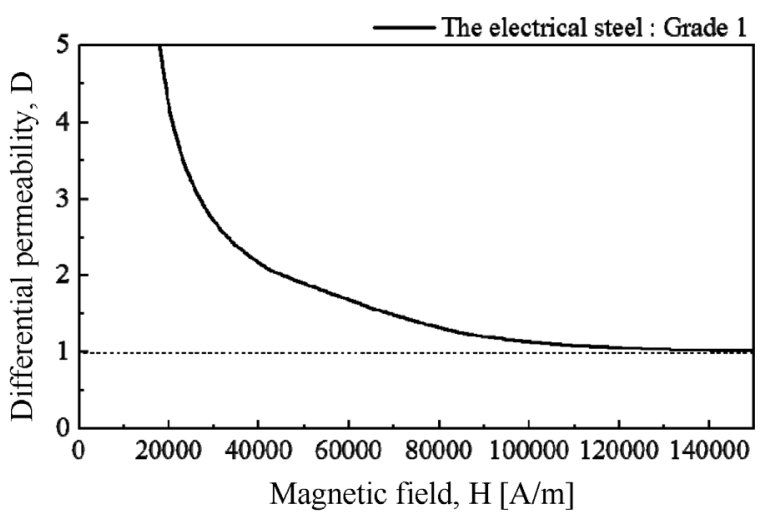

Fig. 6. Differential permeability of B-H curve for the electrical steel, Grade 1.

errors compared to the measured values. Figs. 5 and 6 show the B-H curve and the differential permeability, which are obtained through the development of the quadratic equation for grade 1 among the five test pieces of electrical steel. It is found that the development is smoothly connected to Region II, which is the intermediate area. The saturation magnetic flux density's value of grade 1, calculated from the predicted saturation induction, is $2.163 \mathrm{~T}$, and the experimental value is $2.169 \mathrm{~T}$, which represents only an error rate of $0.3 \%$. Differential permeability is also calculated. In the saturation area, it is
Table 2. Motor parameters.

\begin{tabular}{lc}
\hline \hline & Improved model (unit) \\
\hline Maximum power & Approximately $14 \mathrm{~kW}$ \\
Maximum torque & $128 \mathrm{Nm}$ \\
Rated/Maximum speed & $1160 / 6500 \mathrm{rpm}$ \\
Pole/slot & $16 / 24$ \\
Winding & Concentrated Winding \\
DC link voltage & $320 \mathrm{~V}$ \\
Residual Induction of PM & $1.235 \mathrm{~T}\left(\mathrm{2} 5^{\circ} \mathrm{C}\right)$ \\
& $1.126 \mathrm{~T}\left(@ 100^{\circ} \mathrm{C}\right)$ \\
\hline
\end{tabular}
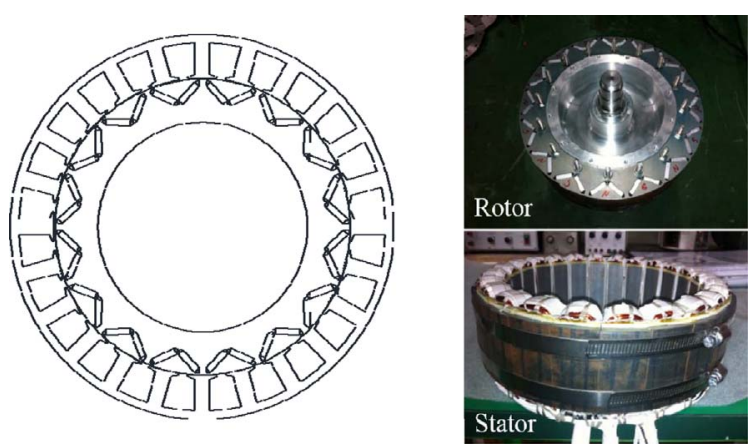

Fig. 7. (Color online) Motor features and motor prototype (rotor and stator assembly).

equal to the value of the permeability of air.

This result confirms that the proposed method for estimating the magnetic saturation induction of the electrical steel is reliable. Therefore, this study is unique because the parameters $\% \mathrm{Al}$ and $\% \mathrm{Si}$ are not required in order to estimate the magnetic saturation induction of the electrical steel, and the latter depends only on the density and resistivity parameters, which can easily be obtained. Furthermore, it is clearly determined that using the extrapolation method with the quadratic equation developed from the saturation induction can be appropriately applied in the design phase.

4.2. Model of electrical machine and analysis method The predicted values of the electromotive force and the motor's torque are estimated by applying B-H curve data 
of the electrical steel grade 1, which is extrapolated and compared to the measured values. In order to verify the accuracy of the comparison, a motor that has a large amount of magnetic leakage flux, and has a severely saturated rib and bridge is selected.

Table 2 presents the specifications of the electrical motor. This is an in-wheel type motor used for vehicle drive. The B-H curve data of the proposed electrical steel are used in the FEA of a rotor with a rib and a bridge. Grade 1 is used to fabricate the motor. Magneto-statics analysis is performed, and motor characteristics such as $\mathrm{BEMF}$ and torque at room temperature $300 \mathrm{~K}\left(26.85^{\circ} \mathrm{C}\right)$ and $373 \mathrm{~K}\left(100^{\circ} \mathrm{C}\right)$ are calculated and compared. In order to ensure the reliability of the proposed method, the calculated characteristics of the electrical motor are compared to those of an actual motor. The full load conditions of the machines are $128 \mathrm{Nm}$ and approximately $14 \mathrm{~kW}$ at a rated speed of $1160 \mathrm{rpm}$, as given in Table 2 .

Based on these assumptions, the electrical motor performance is evaluated in a motor equivalent circuit, considering losses after the motor parameters that reflect the $\mathrm{B}-\mathrm{H}$ data are calculated [6, 13-17].

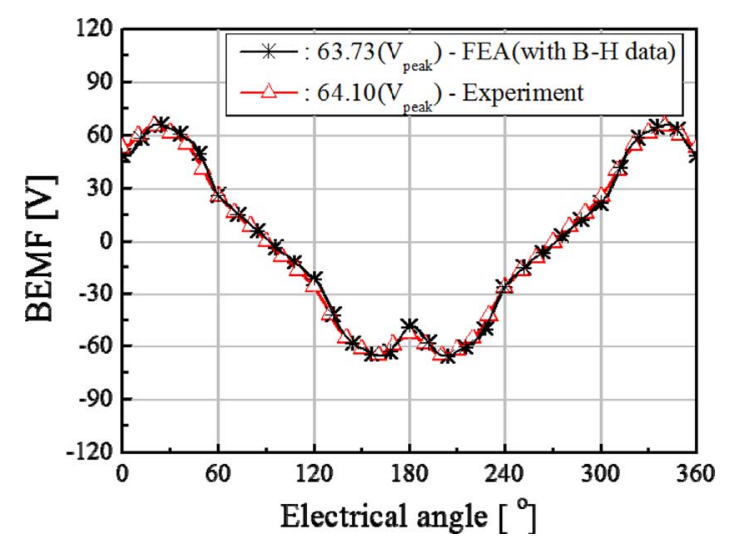

Fig. 8. (Color online) Results for motor characteristics (BEMF).

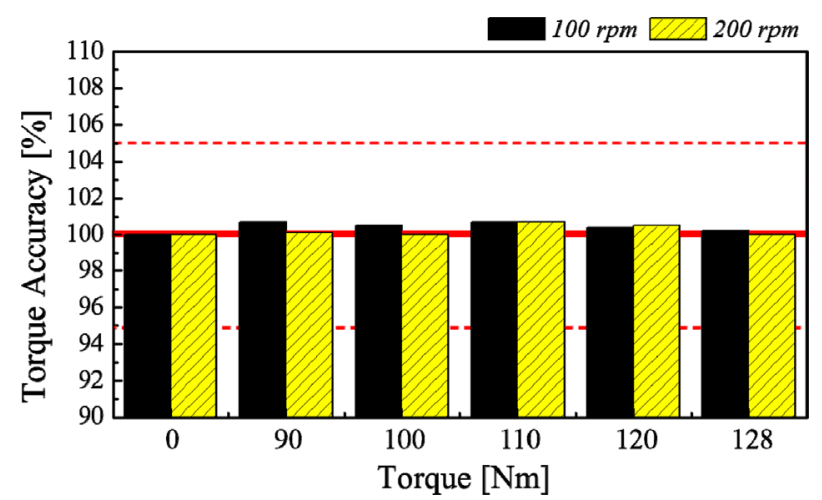

Fig. 9. (Color online) Results for motor characteristics (Torque accuracy).
4.3. Results of the motor characteristics using the extrapolated B-H curve data for FEA

Fig. 8 illustrates the produced motor and its rotor and stator used to validate the proposed electrical B-H curve data. A load machine, a torque sensor, and the test motor are serially and mechanically connected in the experimental setup. Fig. 8 compare the experimental and analytical performance values of the electrical motor, designed using grade 1 steel. The line-to-line BEMF values, at $1000 \mathrm{rpm}$, under the no-load condition, obtained via nonlinear $2 \mathrm{D}$ FEA, are compared to the experimental results. The error is approximately $0.57 \%$, which is generally a satisfying value. Furthermore, the tendencies of the experimental and analytical waveforms in the graph are very similar. Fig. 9 presents the torque accuracy under various load conditions. The error between the experimental and analytical results for the torque is under $0.7 \%$. Thus, applying the $\mathrm{B}-\mathrm{H}$ data obtained by the proposed method in the motor design provides reliable results.

\section{Conclusion}

This paper investigated a novel method to estimate the magnetic saturation induction using only the density and the resistivity values of electrical steel. These parameters can conveniently be obtained through the derivation of a mathematical formula using the $\mathrm{Al}$ and $\mathrm{Si}$ contents (wt\%). In addition, the proposed method to extrapolate the B-H curve using the quadratic equations from the magnetic saturation induction was introduced. The estimation method using the motor material properties was determined through analytical and experimental processes. The extrapolation method was used to investigate the saturation point in electrical steel. The difference between the $J_{s}$ analytical and experimental results is within $1.2 \%$. The results showed that the density and resistivity values of the steel are important factors in estimating the B-H saturation induction. In particular, the saturation induction of electrical steel can be estimated using only the density and resistivity parameters, instead of the $\% \mathrm{Al}$ and $\% \mathrm{Si}$ values. Moreover, the characteristics of the electrical motor can be accurately identified using the extrapolated B-H data. Based on the analytical results, a motor model was fabricated and tested. The simulations and design method were validated through experimental tests. The measured error rate of the electromotive force and the torque were within $0.57 \%$ and $0.7 \%$, respectively. The proposed extrapolation method is undoubtedly useful for accurate estimation of the electrical steel properties and for designing the IPMSMs characteristics, using a finite element method based on the extrapolated B-H data. 


\section{Acknowledgments}

This research was supported by the MSIP (Ministry of Science, ICT and Future Planning), Korea, under the CITRC (Convergence Information Technology Research Center) (IITP-2015-H8601-15-1005) supervised by the IITP (Institute for Information \& communication Technology Promotion).

This work supported by a research program (The Specialized Research Center on the Future Ground System) funded by the Agency for Defense Development of Korea and we appreciate it.

\section{References}

[1] K. I. Laskaris and A. G. Kladas, IEEE Trans. Ind. Electron. 57, 138 (2010).

[2] K. T. Chau, C. C. Chan, and Liu Chunhua, IEEE Trans. Ind. Electron. 55, 2246 (2008).

[3] K. C. Kim, C. S. Jin, and J. Lee, IEEE Trans. Magn. 45, 2835 (2009).

[4] Y. Kano, T. Kosaka, N. Matsui, and M. Fujitsuna, Int. Conf. Electrical Machines 2709 (2012).

[5] K. Fujiwara, T. Adachi, and N. Takahashi, IEEE Trans. Magn. 38, 889 (2002).

[6] T. Takeuchi, 'Electric machine design' (Ohmsha, Ltd.,
1979).

[7] US $7887645 \mathrm{~B} 1$

[8] J. Barros Lorenzo, T. Ros-Yañez, M. De Wulf, and Y. Houbaert, IEEE Trans. Magn. 40, 2739 (2004).

[9] K. Ikeda and H. Dohmeki, IEEE International Conference on Electrical Machines (ICEM), 784 (2012).

[10] T. Yonamine and F. J. G. Landgraf, J. Magn. Magn. Mater. 272-276, e565 (2004).

[11] R. C. Hall, J. Appl. Phys. 31, 1037 (1960).

[12] R. M. Bozorth and D. Ferromagnetism, Van Nostrand Company, Inc., Princeton, NJ (1955).

[13] D. C. Hanselman and W. H. Peake, IEE Proc. Electr. Power Appl. 142, 131 (1995).

[14] B. H. Lee, S. O. Kwon, T. Sun, J. P. Hong, G. H. Lee, and J. Hur, IEEE Trans. Magn. 47, 1066 (2011).

[15] G. H. Kang, J. P. Hong, G. T. Kim, and J. W. Park, IEEE Trans. Magn. 36, 1867 (2000).

[16] M. S. Lim, S. H. Chai, and J. P. Hong, IET Electric Power Applications 8, 349 (2014).

[17] B. H. Lee, S. O. Kwon, T. Sun, J. P. Hong, G. H. Lee, and J. Hur, IEEE Trans. Magn. 47, 1066 (2011).

[18] A. E. Umenei, Y. Melikhov, and D. C. Jiles, IEEE Trans. Magn. 47, 4706 (2011).

[19] R. M. Bozorth, Ferromagnetism, New York, IEEE Press, (1993) p. 476.

[20] M. Jesenik, A. Hamler, P. Kitak, and M. Trlep, Compumag 2013, Budapest (2013). 